\title{
DRAFT
}

Manuscript in print (Fire Safety Journal 43 (2008) 127-139)

\section{Treatment of Design Fire Uncertainty using Quadrature Method of Moments}

\begin{abstract}
The use of a single design fire in a performance based fire design code typically fails to account for the inherent uncertainty in knowledge of the future use of the space. Uncertainties in knowledge of intended use and the implications in terms of fuel loading and potential heat release rate can be bounded using probabilistic methods. Use of a cumulative distribution function (CDF) and the related probability density function (PDF) specify the best available estimate of the probability (likelihood) of a fire of given size to take place in a compartment. Monte Carlo simulation is a widely used computational method for treating uncertainty that might be described by a PDF . In this technique, one samples the uncertain variables from their underlying PDFs and runs a fire model for each sample. For complex fire models, this approach may be computationally intractable. In this work we present a computationally efficient technique called the Quadrature Method of Moments (QMOM) for propagating uncertainty bound in distributions. In QMOM one solves for only the moments of a relevant uncertain parameter. The cumulative distribution function $(\mathrm{CDF})$ of the uncertain parameter provides all the statistical information required for risk assessments. We consider a simplified propagation of uncertainty problem. Results using both the ASET and CFAST fire models indicate that computation of the moments of the PDF using QMOM and the reconstruction of the CDF by matching the moments with those of a 4-parameter Generalized Lambda Distribution (GLD) give accurate results at a significantly smaller computational cost.
\end{abstract}

Keywords: Design fire; uncertainty; Monte Carlo simulation; Quadrature Method of Moments; Generalized Lambda Distribution.

\section{Introduction}

Performance based fire design (PBFD) stipulates that a building must satisfy some performance requirement. That is, the fire safety of the building must be evaluated before the building can be deemed fit for occupancy (e.g. [1]). This is normally done by simulating fire evolution in a structure and evaluating safety criteria, such as the height of the smoke layer at some critical time after the start of the fire. The fire model typically consists of a design fire, i.e. a typical fire with a typical rate of heat release. However, the use of a single design fire while simulating a fire scenario may be inadequate. The type of combustible materials in a room, their arrangement and the point and source of ignition are unpredictable. To account for this variability, the use of a single representative fuel has been proposed in [2]. This is a single fuel source, located at a center of the room, having the same heat release rate characteristics as the actual distribution of combustible material in the room. The single representative fuel source may be considered to be an average of the actual distribution of fuel sources. Due to the extreme variability in the type of fire that can occur in any modern building compartment, a single average 


\section{DRAFT}

representative fuel source may not be sufficient to characterize all the possible fire scenarios, and therefore one may have to consider a distribution of fire sizes. Furthermore, there are many other uncertainties such as operation of safety devices such as smoke detectors and sprinklers, opening or closing of vents etc. An early discussion of the uncertainties inherent in fire safety design is given by [3]. In recent years there have been systematic studies to incorporate the uncertainty inherent in the variables relevant to any given design fire. A thorough discussion of existing techniques is discussed in [4]. In this study, we investigate a very general mathematical technique that can be used to simulate the propagation of uncertainty of any variable that is used in a fire model. To this point our discussion of the use of probabilistic methods for performance based design analysis has been quite abstract. A more physically based example is provided to explore the use of the techniques and the value of the outputs.

Consider a design problem in which a designer requires an active fire protection system if a smoke layer descends below some critical height at a critical time as might be found from an egress model. Clearly, the layer height is strongly dependent on the fire size that would be assumed to take place in the compartment. As previously noted, the designer does not know a-priori the range of use of the compartment. One means of clarifying the likely hazard associated with a range of potential fires is through the use of probabilistic assumptions about the fire size and fire models used to propagate the uncertainty in the fire size into a layer height distribution. The problem is shown schematically in Figure 1. We have a deterministic fire model that takes as input the heat release rate of the fire and provides as output the height of the smoke layer. Any uncertainty in the input variables is propagated in time by the fire model and gives rise to the uncertainty of the output parameter. The uncertainty in input variable, the heat release rate, is represented using a probability distribution function (PDF). For the output variable, the smoke layer height, the cumulative distribution function (CDF) is desired. While the PDF and CDF contain the same statistical information and are directly related by the fundamental theorem of calculus, the CDF is more convenient for displaying useful statistical information that can be used in risk assessment studies, such as the probability of the smoke layer height to be below some critical value at different instances after the occurrence of a fire. The two models that we have considered are the Available Safe Egress Time (ASET) Model [5] and the Consolidated Fire and Smoke Transport (CFAST) model [6]. These are both deterministic fire models that require other input parameters such as the height and area of the enclosure, the location of the vents, windows etc. In this study, these parameters are assumed to be known to a high level of certainty. The only uncertain variable is the heat release rate that is usually sampled from a statistical distribution of known/historical data. Problems of this type in which one has uncertain inputs in a deterministic fire model have been discussed by Magnusson et al. [7]. In the same article, the authors discuss the use of Monte Carlo simulation as an attractive technique for solving these problems. Monte Carlo simulations although easy to use can be extremely costly in terms of computational requirements. These methods work by sampling from the PDF of the input variable and running the fire model for each sample. A large number of random samples may be needed for accurate representation of the PDF. If the fire model is sufficiently complex then each run of the fire model involves considerable computational cost and Monte Carlo simulations become prohibitively 


\section{DRAFT}

expensive. To address these issues, we look at an alternative approach involving the method of moments and reconstruction of the CDF using the moments of the PDF. The discussion of this method follows.

\section{Mathematical representation of the propagation of uncertainty and the quadrature method of moments}

We have a system where the dynamics depends on a random input variable such as the heat release rate. Let $\dot{S}$ be the fire heat release rate, assumed to be stochastic, and let $m(\dot{S})$ be its PDF. The output of the model will be the PDF of the smoke layer height $n(Z)$ at some critical time, $t_{c r}$, where $Z\left(t_{c r}\right)$ is the smoke layer height from the floor at the critical instant. The mathematical representation then consists of the transformation from $m\left(\dot{S}, t=t_{0}\right)$ to $n\left(Z, t=t_{c r}\right)$ which is given by the change in variable rule for PDFs, $m(\dot{S}) d \dot{S}=n(Z) d Z$

The actual mapping between $\dot{S}\left(t=t_{0}\right)$ and $Z\left(t=t_{c r}\right)$ is given by

$Z\left(\dot{S}, t_{c r}\right)=\int_{t_{0}}^{t_{c r}} f(\tau, \dot{S}(\tau)) d \tau$

where $\left\{\begin{array}{l}\frac{d Z}{d t}=f(t, \dot{S}(t)) \\ Z\left(t=t_{0}\right)=Z_{0}\end{array}\right.$ is provided by the fire model discussed later.

A widely used technique for obtaining the statistical properties of the layer height is through Monte Carlo Simulations. In this technique, $\dot{S}$ is randomly sampled form its known distribution and the fire model is integrated for each $\dot{S}$ to build up an ensemble of $Z$. As discussed earlier, this can be a computationally intensive procedure since a large number of samples of $\dot{S}$ need to be taken to obtain good statistics. If the fire model is sufficiently complex, each run of the fire model can be very expensive. In this study we attempt to solve the problem using the method of moments. We seek only the moments of the PDF of $Z, n(Z)$ and then attempt to reconstruct the CDF of $Z$ using the moments. The moments of $n(Z)$ can be written in terms of the initial PDF of $\dot{S}, m(\dot{S})$ using 2.1 and 2.2

$M_{k}^{(Z)}=\int_{Z_{\min }}^{Z_{\max }} Z^{k} n(Z) d Z=\int_{\dot{S}_{\min }}^{\dot{S}_{\max }} Z(\dot{S})^{k} m(\dot{S}) d \dot{S}$

where $M_{k}^{(Z)}$ is the kth moment of $n(Z)$. Hence the problem reduces to the task of finding an accurate approximation of $M_{k}^{(Z)}$ given the moments of the initial distribution $m(\dot{S})$. The approximation is carried out using the Gaussian quadrature rule with unknown weight function that is used in the quadrature method of moments ([8]).

$M_{k}^{(Z)}=\int_{\dot{S}_{\min }}^{\dot{S}_{\max }} Z\left(\dot{S}, t_{c r}\right)^{k} m(\dot{S}) d \dot{S} \approx \sum_{n=1}^{N_{Q}} Z\left(\dot{S}_{n}, t_{c r}\right)^{k} W_{n}$ 


\section{DRAFT}

Here $\dot{S}_{n}$ are the quadrature points and $W_{n}$ are the quadrature weights that are obtained from the moments of $m(\dot{S})$. Further details on how the quadrature points and weights can be computed from the moments can be found in [8]. It is seen that this technique requires only $N_{Q}$ samples of $\dot{S}_{n}$ determined from $2 N_{Q}$ moments of $m(\dot{S})$. The fire model is run for only the $N_{Q}$ heat release rates $\left(\dot{S}_{n}\right)$ to get $Z\left(\dot{S}_{n}, t_{c r}\right)$. If $N_{Q}$ is small, typically 3 or 4 , then this technique enables a dramatic reduction in the computational effort required for these types of problems. There are two computational tasks involved in determining the feasibility of the QMOM approach. Firstly, one needs to determine the accuracy of the moments predicted using QMOM. This is necessary because the set of moments $M_{k}^{(Z)}$ is given by a quadrature approximation using a limited number of quadrature points, $N_{Q}$, and one needs to find the optimum $N_{Q}$ that gives accurate moments. Secondly, the moments do not give all the information that is contained in a CDF. Therefore, the CDF needs to be reconstructed from a finite number of known moments. This is carried out by matching the calculated moments to the moments of a fourparameter distribution. A large number of distributions are available that can be used for CDF reconstruction. In this work we follow the method outlined in [9]. As discussed later, we find that the Generalized Lambda Distribution (GLD) is best suited for reconstruction of the CDFs that we obtain from our computational models. A schematic of the methodology is provided in Fig. 2.

\section{Details of input variable distributions and fire models used}

The methodology outlined in Fig. 1 shows that the computational model requires an input PDF of heat release rates and a fire model for evaluating the output parameter for each heat release rate.

\subsection{Input heat release rate distribution}

We use the generalized beta distribution for the input heat release rate PDF. In practice, the PDF needs to be determined from empirical or historical data. The generalized beta PDF is a four parameter distribution. It is very versatile because one can create different shaped PDFs by varying the four parameters. Further the beta PDF has bounded support which means that one can specify the maximum and minimum fire sizes that can occur in a compartment. This feature prevents the possibility of unrealistically large fires in a room of finite size containing a finite amount of combustible material. The generalized beta distribution with parameters $\beta_{1}, \beta_{2}, \beta_{3}, \beta_{4}$ is given by [9].

$$
m(\dot{S})=\left\{\begin{array}{l}
\frac{\left(\dot{S}-\beta_{1}\right)^{\beta_{3}}\left(\beta_{1}+\beta_{2}-\dot{S}\right)^{\beta_{4}}}{\beta\left(\beta_{3}+1, \beta_{4}+1\right) \beta_{2}^{\left(\beta_{3}+\beta_{4}+1\right)}} ; \text { for } \beta_{1} \leq \dot{S} \leq \beta_{1}+\beta_{2} \\
0 ; \text { otherwise }
\end{array}\right.
$$

Here $\beta_{1}, \beta_{2}$ are the location and scale parameters. $\beta$ is the beta function. The beta function can be defined in terms of the Gamma function, $\Gamma$, that is more widely available: 


\section{DRAFT}

$\beta(x, y)=\frac{\Gamma(x) \Gamma(y)}{\Gamma(x+y)}$

We have taken $\beta_{1}=0$ and $\beta_{2}=200$. This choice defines the range of fire sizes to be between 0 and $200 \mathrm{~kW} . \beta_{3}$ and $\beta_{4}$ are the shape parameters. Different shaped beta PDFs can be obtained by changing $\beta_{3}$ and $\beta_{4}$. The four different PDFs we use are defined by:

I. $\beta_{3}=1 ; \beta_{4}=4$

II. $\beta_{3}=0 ; \beta_{4}=2$

III. $\beta_{3}=2 ; \beta_{4}=0$

IV. $\beta_{3}=1 ; \beta_{4}=1$

For all these cases $\beta_{1}=0$ and $\beta_{2}=200$. Fig. 3 shows the different PDFs. PDFs II and III may respectively model situations where small and large sized fires are more likely to occur respectively. I and II model situations where mid sized fires may be more likely. Next, we consider the fire models used.

\subsection{Available Safe Egress Time (ASET) Model}

We use two well characterized zone models for simulating compartment fires. The first model is the Available Safe Egress Time Model (ASET) that is described in detail in [5]. The governing equation for the smoke layer height is

$\frac{d Z}{d t}=-\frac{\dot{m}_{e}}{\rho_{a} A}-\frac{\left(1-L_{c}\right) \dot{S}}{\rho_{a} c_{p} T_{a} A}$

$\dot{m}_{e}$ is the rate of entrainment of the air into the plume and is given by a correlation for plume flow.

$\dot{m}_{e}=0.21 \rho_{a}(g \Delta Z)^{1 / 2} \Delta Z^{2}\left[\frac{\left(1-L_{r}\right) \dot{S}}{\rho_{a} C_{a} T_{a}(g \Delta Z)^{1 / 2} \Delta Z^{2}}\right]^{1 / 3}$

The properties of air are density $\left(\rho_{a}=1 \mathrm{~kg} / \mathrm{m}^{3}\right)$ and specific heat capacity $\left(c_{a}=1.004\right.$ $\mathrm{kJ} / \mathrm{kgK}) . L_{c}$ and $L_{r}$ are empirical constants taken to be 0.8 and 0.35 respectively. The ambient temperature is $\left(T_{a}=300 \mathrm{~K}\right) . g=9.8 \mathrm{~m} / \mathrm{s}^{2}$ is the acceleration due to gravity. The floor area $A$ is $31.5 \mathrm{~m}^{2}, \Delta Z=Z-Z_{f}$ where $Z_{f}=0.2 \mathrm{~m}$ is the height of the base of the fire and the initial condition $Z\left(t=t_{0}\right)$ is the ceiling height which is taken as $6.15 \mathrm{~m} . \dot{S}$ is the heat release rate which is random and whose PDF is given by the generalized beta distribution. We assume that $\dot{S}$ does not change with time. The range of fire heat release rates, room geometry, empirical constants and the assumption of steady heat release rate correspond to the conditions used in the experimental validation of the ASET model reported in [10].

\subsection{Consolidated Fire and Smoke Transport (CFAST) Model}

CFAST is a more sophisticated zone model than ASET that is widely used by architects, fire protection engineers, safety officials etc. [6]. It is a two zone model that 


\section{DRAFT}

models the evolution of smoke, combustion gases and temperature in a building compartment that is on fire [11]. The details of the software program can be found in reference [11]. One can take CFAST to be a fire model that provides, among other things, the smoke layer height for a particular fire heat release rate. CFAST simulations are performed for two cases. In one case we use exactly the same compartment geometry as in ASET. In the second case, we add a window of width $4 \mathrm{~m}$, height $2 \mathrm{~m}$ located $2 \mathrm{~m}$ above the floor as shown in Fig. 4.

\section{Computational methods}

\subsection{Monte Carlo Simulations}

In the Monte Carlo simulations, the fire heat release rates, $\dot{S}$, are sampled from the generalized beta distribution (3.1) using the rejection sampling method discussed in [12]. ASET and CFAST models are run for each sample of $\dot{S}$ as an input. These models return the smoke layer height, $Z$, at a specified critical time. We have arbitrarily chosen the critical time to be $120 \mathrm{~s}$. The CDF of $\mathrm{Z}$ can then be directly obtained from the output of the multiple runs of the fire models. More efficient methods of sampling from a PDF have been developed (e.g. as discussed in [7]). Our objective in performing Monte Carlo simulations is to determine the accuracy of the results obtained using moment methods and therefore we do not consider issues on the computational efficiency of our Monte Carlo simulations.

\subsection{Quadrature Method of Moments (QMOM)}

The quadrature method of moments is a very general technique for solution of equations involving the dynamics of a PDF [8], [13], [14]. In this work we discuss its application to an uncertainty propagation problem discussed in section 2. In QMOM, one first computes the moments of the beta distribution, $m(\dot{S})$, given in 3.1. The moments of the beta distribution are given by [9]:

$M_{k}=E\left(\dot{S}^{k}\right)=\frac{\beta\left(\beta_{3}+k+1, \beta_{4}+1\right)}{\beta\left(\beta_{3}+1, \beta_{4}+1\right)}$

$M_{k}$ is the kth moment of the beta distribution, $\beta$ is the beta function and $\beta_{3}, \beta_{4}$ are the shape parameters. Using a sequence of $2 N_{Q}$ moments, one can find $N_{Q}$ quadrature points and weights using the method outlined in [8]. This method essentially involves computing the eigenvalues and eigenvectors of a tri-diagonal matrix whose elements can be computed in terms of the moments. A very efficient algorithm, called the Product Difference Algorithm, exists for computing the tri-diagonal matrix from a given moment sequence. Details of the method can be found in [8] or [13]. The $N_{Q}$ quadrature points $\dot{S}_{n, B D}$ correspond to the beta PDF. The corresponding quadrature points for the generalized beta distribution (GBD) can be obtained using

$\dot{S}_{n, G B D}=\dot{S}_{n, B D} \cdot \beta_{2}+\beta_{1}$ 


\section{DRAFT}

where $\dot{S}_{n, G B D}$ is the quadrature point for the GBD. $\beta_{1}$ is the location parameter $\beta_{2}$ the scale parameter defined earlier. In QMOM, one can choose either an integral moment sequence such as $\left\{M_{0}, M_{1}, M_{2}, \ldots\right\}$ or any fractional moment sequence such as $\left\{M_{k / L}\right\}=\left\{M_{0}, M_{1 / L}, M_{2 / L}, \ldots\right\}$. Upadhyay and Ezekoye [15] have shown that the use of a fractional moment sequence can sometimes lead to better quadrature approximations. The theoretical details and methods for using fractional moment sequences for QMOM applications can be found in [15].

For the moment method, the $N_{Q}$ quadrature points can be considered to be the samples of the heat release rate. The fire model is then run for each of these sampled heat release rates to get $N_{Q}$ smoke layer heights at a specified instant. Therefore, the QMOM technique can be considered to be an efficient way of sampling from a known PDF. The moments of the layer height can be obtained using equation 2.4. The CDF is then reconstructed by matching the computed moments to the moments of the four-parameter GLD.

QMOM can be used as an alternative to Monte Carlo simulations in many applications. To the best of the authors' knowledge, there have not been many studies comparing the computational cost and accuracy of QMOM with Monte Carlo Simulations. A rare example is [16] where comparisons of QMOM with Monte Carlo simulations for crystal growth and aggregation are carried out. The crystal growth problem is mathematically similar to the statistical uncertainty problem considered in this work. In [16] the authors demonstrate very low percentage errors for both the lower and higher order moments obtained using QMOM.

\subsection{GLD reconstruction of the $C D F$}

Karian and Dudewicz [9] detail the basis and development of the Generalized Lambda Distribution (GLD) for use in fitting statistical data. The four parameter GLD can be represented by $\operatorname{GLD}\left(\lambda_{1}, \lambda_{2}, \lambda_{3}, \lambda_{4}\right)$. The GLD is most easily specified in terms of the percentile function

$Q(y)=Q\left(y ; \lambda_{1}, \lambda_{2}, \lambda_{3}, \lambda_{4}\right)=\lambda_{1}+\frac{y^{\lambda_{3}}-(1-y)^{\lambda_{4}}}{\lambda_{2}}$

with $0 \leq y \leq 1$. The $\mathrm{CDF}$ is obtained as an inverse of 4.4, i.e. $C D F(Q(y))=y$. The central task of the GLD method is to obtain the four parameters $\lambda_{1}, \lambda_{2}, \lambda_{3}, \lambda_{4}$ from the four moments $\left(M_{k}^{(Z)} ; k=1,2,3,4\right)$ of equation 2.4. One computes the skewness $\alpha_{3}$ and kurtosis $\alpha_{4}$ from the raw moments. One can also compute these terms for the GLD distribution using the (unknown) parameters $\lambda_{3}$ and $\lambda_{4}$.

$$
\begin{aligned}
& \alpha_{3}=\frac{M_{3}-3 M_{1} M_{2}+2 M_{1}^{3}}{\left(M_{2}-M_{1}{ }^{2}\right)^{3 / 2}}=G_{3}\left(\lambda_{3}, \lambda_{4}\right) \\
& \alpha_{4}=\frac{M_{4}-4 M_{1} M_{3}+6 M_{1}^{2} M_{2}-3 M_{1}^{4}}{\left(M_{2}-M_{1}{ }^{2}\right)^{2}}=G_{4}\left(\lambda_{3}, \lambda_{4}\right)
\end{aligned}
$$




\section{DRAFT}

The $G_{i}$ are irrational functions of $\lambda_{3}$ and $\lambda_{4}$ and contain beta functions in $\lambda_{3}$ and $\lambda_{4}$. The difficulty lies in solving the coupled, nonlinear, bivariate equations in 4.5 to get an optimum $\lambda_{3}, \lambda_{4}$. In this study we simply use the results for $\lambda_{3}$ and $\lambda_{4}$ presented in tabular form in [9]. The table provides $\lambda_{3}$ and $\lambda_{4}$ along with $\lambda_{1}(0,1), \lambda_{2}(0,1)$ for a wide range of allowable values of the skewness and kurtosis $\left(\alpha_{3}\right.$ and $\left.\alpha_{4}\right)$. Linear interpolation is used for values in between. There are spaces of $\left(\alpha_{3}, \alpha_{4}\right)$ that are not covered by the tables. In some of these cases a nonlinear optimization problem must be solved for equation 4.5, while for other cases either the generalized beta distribution (GBD) is a better fit or else the reconstruction of the CDF using GLD/GBD is impossible. In all our simulations, $\left(\alpha_{3}, \alpha_{4}\right)$ fell in the range covered by the tables. $\lambda_{1}$ and $\lambda_{2}$ can be computed from the tabulated $\lambda_{1}(0,1), \lambda_{2}(0,1)$ using

$\lambda_{1}=\lambda_{1}(0,1) \sqrt{\alpha_{2}}+\alpha_{1} ; \lambda_{2}=\lambda_{2}(0,1) / \sqrt{\alpha_{2}}$

where $\alpha_{2}=M_{2}-M_{1}^{2}$ is the variance and $\alpha_{1}=M_{1}$ is the mean of the smoke layer height distribution obtained from the QMOM solutions. The four lambdas give us the percentile function, $Q(y)$, whose inverse gives the CDF.

\section{Results and Discussions}

\subsection{Comparison of ASET and CFAST results}

We first compare results obtained using CFAST and ASET results. The room geometry is the same as used by Hurley [10] for comparison of the ASET predictions of the smoke layer height with full-scale test data. We take two particular cases with constant fire heat release rates of $195 \mathrm{~kW}$ and $33 \mathrm{~kW}$ (test \#1 and test\#5 in [10]). These cases correspond to some of the tests in which a constant heat release rate was maintained and these heat release rates also fall within the range that we consider in this study. Fig. 5 shows the comparison of ASET and CFAST predictions of the smoke layer height for two different heat release rates. ASET results closely match the test data. These results agree with those presented by Hurley [10]. However, the CFAST results show smaller layer heights for both heat release rates.

\subsection{Monte Carlo Simulation results for ASET and CFAST models}

Fig. 6 shows the PDF of the smoke layer height obtained from Monte Carlo simulations using the ASET model. Four different PDFs of the smoke layer, $n(Z)$, are obtained for the four different input heat release rate PDFs, $m(\dot{S})$, described in 3.1. 10,000 Monte Carlo samples of the heat release rates are taken to ensure converged results. All the pdfs show positive skewness and kurtosis (i.e. they are asymmetric, have a higher peak around the mean and fatter tails compared to a normal distribution). The mean smoke layer height depends on the $m(\dot{S})$. For instance, PDF III consists of a distribution of larger heat release rate fires and consequently the smoke layer heights are smaller. PDF II consists of a distribution of smaller heat release rate fires and the smoke layer heights are larger (farther from the ground). Fig. 7 shows the corresponding CDFs. 


\section{DRAFT}

The CDFs are more useful for risk assessments since the probability of the layer being below any given value is immediately available from the CDF. Despite the variability in the shapes of the PDFs, the CDFs are almost of the same shape, only the locations where they peak are different. Fig. 8 shows the CDF obtained using Monte Carlo simulations for the CFAST model where the compartment is exactly the same as for the ASET model. Fig. 9 shows the CDFs obtained using CFAST model for a compartment with a window (Fig. 4 shows the compartment geometry for both cases). PDFs for the CFAST models show the same qualitative features as the ASET model. As discussed in section 5.1, smoke layer heights predicted using CFAST are smaller than those predicted by ASET. Differences in the CDFs due to the presence of a window are also apparent. Since the window allows smoke to escape, we see that smoke layer height peaks between 2.5 and 3 m. Due to increased complexity of the CFAST models, only 500 samples are used for the heat release rate. Furthermore, the smallest resolution of the smoke layer heights output by CFAST is $10 \mathrm{~cm}$. For these reasons, both the PDFs and CDFs obtained using CFAST are coarser compared to those obtained using ASET.

\subsection{QMOM results for ASET and CFAST models}

In the quadrature method of moments, one obtains the moments of the smoke layer height PDF, $n(Z)$. The moments required for matching with the GLD moments are the mean, variance, skewness and kurtosis. Since the moments of $\mathrm{n}(\mathrm{Z}), M_{k}^{(Z)}$, are obtained from a quadrature approximation, it is essential to determine the accuracy in their prediction. As shown in equation 4.5, the skewness and kurtosis are functions of the moments $M_{k}^{(Z)}$. They are important quantities because the GLD parameters $\lambda_{3}, \lambda_{4}$ are found by matching the skewness and kurtosis (equation 4.5). In Fig. 10 and Fig. 11, we compare the skewness and kurtosis predicted using QMOM with those obtained from converged Monte Carlo simulations for the ASET model. We see that both skewness and kurtosis fail to converge for the choice of an integral moment set (e.g. $\left\{M_{0}, M_{1}, M_{2} \ldots\right\}$ ). However the choice of fractional moment sets such as the $\mathrm{k} / 2, \mathrm{k} / 3$ and $\mathrm{k} / 4$ sets (i.e. the sets $\left.\left\{M_{0}, M_{1 / 2}, M_{1}, M_{3 / 2}, \ldots\right\},\left\{M_{0}, M_{1 / 3}, M_{2 / 3}, M_{1}, \ldots\right\},\left\{M_{0}, M_{1 / 4}, M_{2 / 4}, M_{3 / 4}, \ldots\right\}\right)$, gives faster convergence and more accurate predictions. For instance, just three quadrature (or sampling) points determined from a set of $6 \mathrm{k} / 4$ moments gives very accurate results for all cases considered. Similar trends are seen for the prediction of means and variances although their predictions are much more accurate.

Fig. 12 shows the reconstructed CDFs using the Generalized Lambda Distribution (GLD) compared with CDFs obtained from Monte Carlo simulations. To get the moments, $M_{k}^{(Z)}$, four quadrature points, $\dot{S}_{n, G B D}$, obtained from eight k/4 moments of $m(\dot{S})$ are used in all cases. The GLD parameters are obtained from appendix B in Karian and Dudewicz (2000). Fig. 12 shows that the GLD reconstruction is very accurate for all the four CDFs. CDFs are sometimes also plotted on probability paper ([17]). In specially designed probability paper or graph, the dependent state variable and the CDF are both rescaled to produce a linear plot. For the GLD, it is not possible to analytically derive a linear relation between the appropriately rescaled variables. We therefore create an arbitrary scale such that the relation between $\operatorname{CDF}(\mathrm{Z})$ and $\mathrm{Z}$ is linear with slope of unity. 


\section{DRAFT}

Fig. 13 shows the CDF for case I plotted in the rescaled Z-axis. Comparison with Monte Carlo simulation results confirms that the fit is accurate in the entire range including the extremes. Reasonably accurate fits were found for all the other CDFs shown in fig. 12 Fig. 14 shows the comparisons for the CFAST model, with and without a window. It is seen that the GLD reconstruction is accurate for the CFAST model as well. Only one input PDF (PDF I) is shown, but the same effect is seen for all four PDFs.

\section{Conclusions and further work}

As computational models become more widely used for fire safety designs, a methodology for incorporating the uncertainties in any fire scenario becomes extremely important. Due to the extreme complexity of the physical phenomena involved, the occurrence of fire in any building and the chain of events triggered by the fire are very uncertain. Designers and fire safety engineers need efficient computational techniques that provide statistical information for assessing the risk in any fire event. The quadrature method of moments proposed in this work is a general technique that has already been used in many other disciplines to solve for the time and space evolution of the moments of a PDF. In many cases, one finds that QMOM solutions are much more efficient and reasonably accurate when compared to results obtained using Monte Carlo simulations or other solution techniques ([13], [14], [15], [16]).

In this work, we consider the evolution of the smoke layer height given the uncertainties in the fire heat release rate. For this problem, we see that the moments of the smoke layer height PDF can be accurately predicted using very few quadrature points, especially if fractional moments are used to obtain quadrature points and weights. Since each quadrature point acts like a sample for the heat release rate, this method also has the potential to reduce the computational effort in Monte Carlo simulations. Furthermore, the $\mathrm{CDF}$ is reconstructed from the moments by matching the moments with those of a four parameter GLD. For all cases considered, we see that the reconstruction is accurate. The fire models used in this study are all reasonably simple and computational costs for running any given scenario are reasonable. If these zone fire models were replaced by computational fluid dynamics based models, it would be prohibitively expensive to perform Monte Carlo type simulations in order to identify probabilistic estimates of risk. On the other hand, it would be feasible to run several (six to eight) CFD simulations in order to generate an accurate cumulative distribution function of the output variable.

While the results from the present study appear promising, this method remains to be tested for significantly more complex fire scenarios. As discussed earlier, there are a large number of uncertain variables in any fire event and one has to simulate the interactions among all these uncertain variables. While the QMOM is well suited for describing the dynamics of the moments of a univariate PDF (for a single uncertain variable), the extension to multivariate PDFs (for multiple uncertain variables) is not straightforward. There exists an alternate, related technique called the direct quadrature method of moments (DQMOM) [18] that can be used to simulate the dynamics of the moments of a multivariate PDF. For multivariate PDFs, there will be a question on the ability to generate meaningful CDFs for the system. While the QMOM approach appears to suitable for the type of problem considered in this work, both QMOM and DQMOM 


\section{DRAFT}

need to be tested on a number of relevant problems in fire science and engineering to investigate their computational efficiency and accuracy.

\section{Appendix}

Here we present in detail the simplified equations that can be used to calculate the quadrature points and weights as well as the GLD parameters for the case of a six moment (three quadrature point) calculation. We use a three-point (six moments) based integration of the $\mathrm{Z}$ moments. As previously noted, this requires that we calculate six moments of the heat release rate distribution. We consider the example of the Beta Distribution as in the text. The moments of the Beta Distribution as given in equation 4.1 is

$$
M_{k}=\frac{\beta\left(\beta_{3}+k+1, \beta_{4}+1\right)}{\beta\left(\beta_{3}+1, \beta_{4}+1\right)}
$$

We take the case of II with $\beta_{1}=0, \beta_{2}=200, \beta_{3}=0, \beta_{4}=2$ and case II with $\beta_{1}=0, \beta_{2}=200, \beta_{3}=2, \beta_{4}=0$. The zeroth moment is 1 and the other 5 moments (for the 3 point quadrature scheme) are as below.

\begin{tabular}{|l|l|l|l|l|l|l|}
\hline$\beta_{3}$ & $\beta_{4}$ & $\mathrm{M}_{1}$ & $\mathrm{M}_{2}$ & $\mathrm{M}_{3}$ & $\mathrm{M}_{4}$ & $\mathrm{M}_{5}$ \\
\hline 0 & 2 & 0.25 & 0.1 & $5.0 \times 10^{-2}$ & $2.9 \times 10^{-2}$ & $1.8 \times 10^{-2}$ \\
\hline 2 & 0 & 0.75 & 0.6 & 0.5 & 0.43 & 0.38 \\
\hline
\end{tabular}

Quadrature points, $S_{n}$, for the heat release rate distribution are found using a cubic equation solution that depends on the six moments in a relatively complicated manner.

$$
\begin{aligned}
& S^{3}-\left(\frac{M_{5}-A M_{4}+B M_{3}}{M_{4}-2 A M_{3}+\left(2 B+A^{2}\right) M_{2}-2 A B M_{1}+B^{2}}\right) S^{2}+ \\
& {\left[\frac{\left(M_{5}-A M_{4}+B M_{3}\right) A}{M_{4}-2 A M_{3}+\left(2 B+A^{2}\right) M_{2}-2 A B M_{1}+B^{2}}-\frac{M_{4}-M_{1} M_{3}}{M_{2}-M_{1}^{2}}\right] S} \\
& +\frac{\left(M_{4}-M_{1} M_{3}\right) M_{1}}{M_{2}-M_{1}^{2}}-\frac{\left(M_{5}-A M_{4}+B M_{3}\right) B}{M_{4}-2 A M_{3}+\left(2 B+A^{2}\right) M_{2}-2 A B M_{1}+B^{2}}-M_{3}=0
\end{aligned}
$$

The constants $\mathrm{A}$ and $\mathrm{B}$ are given by:

$$
A=\frac{M_{3}-M_{1} M_{2}}{M_{2}-M_{1}^{2}} \text { and } B=\frac{M_{3} M_{1}-M_{2}^{2}}{M_{2}-M_{1}^{2}}
$$

The quadrature weights, $\mathrm{W}_{\mathrm{n}}$, are specified to be:

$$
\begin{aligned}
& W_{1}=\frac{M_{2}-M_{1}\left(S_{3}+S_{2}\right)+S_{2} S_{3}}{\left(S_{1}-S_{2}\right)\left(S_{1}-S_{3}\right)}, W_{2}=\frac{M_{2}-M_{1}\left(S_{3}+S_{1}\right)+S_{1} S_{3}}{\left(S_{2}-S_{1}\right)\left(S_{2}-S_{3}\right)}, \text { and } \\
& W_{3}=\frac{M_{2}-M_{1}\left(S_{1}+S_{2}\right)+S_{2} S_{1}}{\left(S_{3}-S_{2}\right)\left(S_{3}-S_{1}\right)}
\end{aligned}
$$




\begin{tabular}{|l|l|l|l|l|l|l|l|}
\hline$\beta_{3}$ & $\beta_{4}$ & $\mathrm{~S}_{1}(\mathrm{~kW})$ & $\mathrm{S}_{2}(\mathrm{~kW})$ & $\mathrm{S}_{3}(\mathrm{~kW})$ & $\mathrm{W}_{1}$ & $\mathrm{~W}_{2}$ & $\mathrm{~W}_{3}$ \\
\hline 0 & 2 & 14.6 & 69.4 & 141.0 & 0.47 & 0.44 & 0.08 \\
\hline 2 & 0 & 59.0 & 130.6 & 185.4 & 0.09 & 0.44 & 0.47 \\
\hline
\end{tabular}

Note that while the quadrature points (heat release rate samples) reflect the nature of the distribution. The PDF in Case I peaks at lower heat release rates and consequently the lower heat release rate samples are given greater weight. The opposite is true for the PDF in Case IV which peaks at higher heat release rates. Note that this technique is convenient only upto three point quadrature since simple expressions exist for the roots of quadratic and cubic polynomials. For higher order quadrature schemes, the more general technique outlined in [8] is simpler.

To calculate the moments of the $\mathrm{Z}$ distribution simply requires that we solve the fire model with the heat release rate at three values: $S_{1}, S_{2}$, and $S_{3}$. Given the moments of the $\mathrm{Z}$ distribution as specified by equation 2.4 , a CDF from the moments of the $\mathrm{Z}$ distribution is calculated using the GLD. First, the raw moments of the $\mathrm{Z}$ distribution must be converted to centered moments.

$$
\begin{aligned}
& \mu=\int_{Z \min }^{Z \max } Z n(Z) d Z=M_{1} \\
& \sigma^{2}=\int_{Z \min }^{Z \max }(Z-\mu)^{2} n(Z) d Z=M_{2}-\mu^{2} \\
& \alpha_{3}=\frac{\int_{Z \min }^{Z \max }(Z-\mu)^{3} n(Z) d Z}{\sigma^{3}}=\frac{1}{\sigma^{3}}\left(M_{3}-3 \mu M_{2}+2 \mu^{3}\right) \\
& \alpha_{4}=\frac{\int_{Z \min }^{Z \max }(Z-\mu)^{4} n(Z) d Z}{\sigma^{4}}=\frac{1}{\sigma^{4}}\left(M_{4}-4 \mu M_{3}+6 \mu^{2} M_{2}-3 \mu^{4}\right)
\end{aligned}
$$

The GLD parameters are then found as discussed in Section 4.3.

\section{References}

[1] Buchanan AH, Implementation of performance-based fire codes. Fire Safety J 1999;32:377-383.

[2] Yung DT, Benichou N, How design fires can be used in fire hazard analysis. Fire Technol. 2002;38:231-242.

[3] Watts JM, Dealing with uncertainty: some applications in fire protection engineering. Fire Safety J 1986;11(1-2):127-134.

[4] Notarianni KA. Uncertainty. In: DiNenno PJ, Drysdale D, Beyler CL, Walton WD, editors. NFPA HFPE-02; SFPE Handbook of Fire Protection Engineering. $3^{\text {rd }}$ ed., Chapter 4, Section 5. Quincy, MA, 2002, pp. 5-40:5-64.

[5] Janssens ML. An introduction to mathematical fire modeling, $2^{\text {nd }}$ ed. Lancaster, Technomic publishing company inc.,2000. 


\section{DRAFT}

[6] Jones WW, Peacock RD, Forney GP, Reneke PA. CFAST- Consolidated Model of Fire Growth and Smoke Transport (Version 6). Technical reference guide. NIST Special Publication 1026, US Department of Commerce, NIST-BFRL, Gaithersburg, MD, December 2005.

[7] Magnusson SE, Frantzich H, Harada K, Fire safety designs based on calculations: uncertainty analysis and safety verification. Fire Safety J 1996;27:305-334.

[8] McGraw R, Description of aerosol dynamics by the quadrature method of moments. Aerosol Sci. Technol. 1997;27:255-265.

[9] Karian ZA, Dudewicz EJ. Fitting statistical distributions: the generalized lambda distribution and generalized bootstrap methods. Boca Raton, CRC Press, 2000.

[10] Hurley MJ, ASET-B: Comparison of model predictions with full scale test data. J. Fire Prot. Eng. 2003;13:37-63.

[11] Jones, W. W.; Peacock, R. D.; Forney, G. P.; Reneke, P. A. CFAST, Consolidated Model of Fire Growth and Smoke Transport (Version 5). Technical Reference Guide. NIST SP 1030; 153 p. October 2004.

[12] Cheng $\mathrm{RCH}$, Generating beta variates with nonintegral shape parameters. Commun. ACM 1978;21(4):317-322.

[13] Upadhyay RR, Ezekoye OA, Evaluation of the 1-point quadrature approximation in QMOM for combined aerosol growth laws. J. Aerosol Sci 2003;34:1665-1683.

[14] McGraw R, Wright DL, Chemically resolved aerosol dynamics for internal mixtures by the quadrature method of moments. J. Aerosol Sci 2003;34:189-209.

[15] Upadhyay RR, Ezekoye OA, Treatment of size-dependent aerosol transport processes using quadrature based moment methods. J. Aerosol Sci 2003;34:1665-1683.

[16] Marchisio DL, Pikturna JT, Fox, RO, Vigil RD, Barresi, AA. Quadrature method of moments for population balance equations. AIChE J. 2003; 49(5): 1266-1276.

[17] Selwyn EWH, Note on the use of probability paper. Proc. Phys. Soc. 1938; 50: 914918.

[18] Marchisio DL, Fox RO, Solution of population balance equations using the direct quadrature method of moments. J. Aerosol Sci 2005;36:43-73.

\section{Figure Captions}

Fig. 1. Schematic of the propagation of statistical uncertainty problem as applied to a design fire application.

Fig. 2. Schematic illustrating the sampling procedure used in the quadrature method of moments and the procedure for getting the CDF. Note that the schematic shows three quadrature points and three weights which would correspond to six known moments.

Fig. 3. Generalized beta probability density functions (PDFs) used for the fire heat release rates.

Fig. 4. (Left) Compartment geometry for the ASET and CFAST models. (Right) Compartment geometry for the CFAST model with a window. 
Fig. 5. Comparison of the ASET and CFAST model results for the smoke layer height with experimental data reported in [10].

Fig. 6. PDFs of the smoke layer height at a critical time $t_{c r}=120 \mathrm{~s}$ obtained from Monte Carlo Simulations using the ASET model. The labels I, II, III, IV correspond to the different heat release rate PDFs in Fig. 3.

Fig. 7. CDFs corresponding to the PDFs in Fig. 6.

Fig. 8. CDFs of the smoke layer height at a critical time $t_{c r}=120 \mathrm{~s}$ obtained from Monte Carlo Simulations using the CFAST model with the same geometry as for the ASET. I, II, III, IV correspond to the different heat release rate PDFs in Fig. 3.

Fig. 9. CDFs of the smoke layer height at a critical time $t_{c r}=120 \mathrm{~s}$ obtained from Monte Carlo Simulations using the CFAST model with a horizontal vent (window) shown in Fig. 6. I, II, III, IV correspond to the different heat release rate PDFs in Fig. 3.

Fig. 10. Study of the convergence of the skewness with increasing number of quadrature points. Results of using different moment sequences are shown.

Fig. 11. Study of the convergence of the kurtosis with increasing number of quadrature points. Results of using different moment sequences are shown.

Fig. 12. Reconstructed CDF using the Generalized Lambda Distribution (GLD) compared with CDF obtained from Monte Carlo simulations for the ASET model.

Fig. 13. CDF for Case I in figure 12 plotted in "probability paper".

Fig. 14. Reconstructed CDF using the GLD compared with the CDF obtained from Monte Carlo Simulations using the CFAST model, with and without a window. The CDFs obtained using the ASET model is also shown. PDF I is used for the heat release rate PDF. 


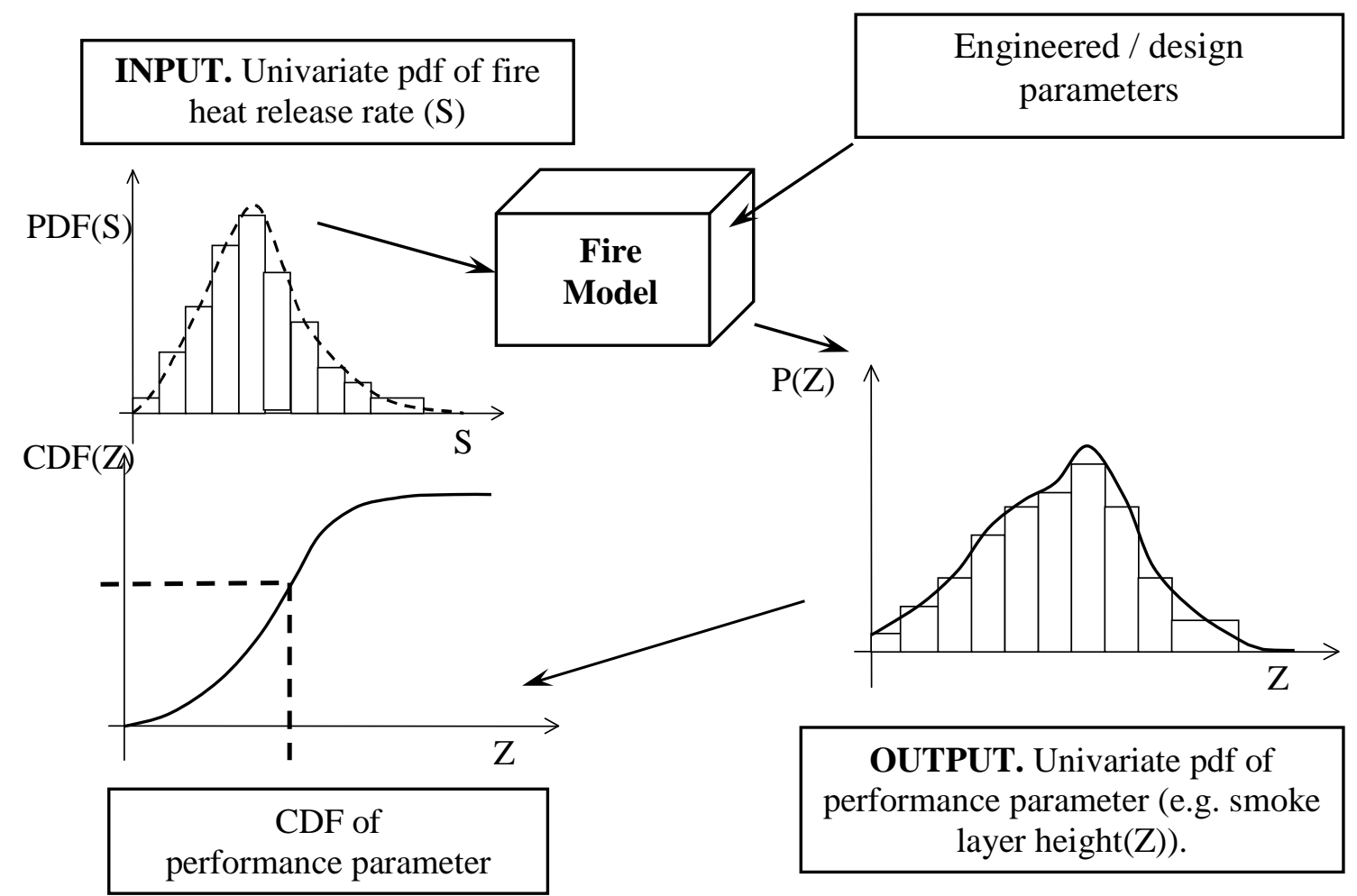

Fig. 1.

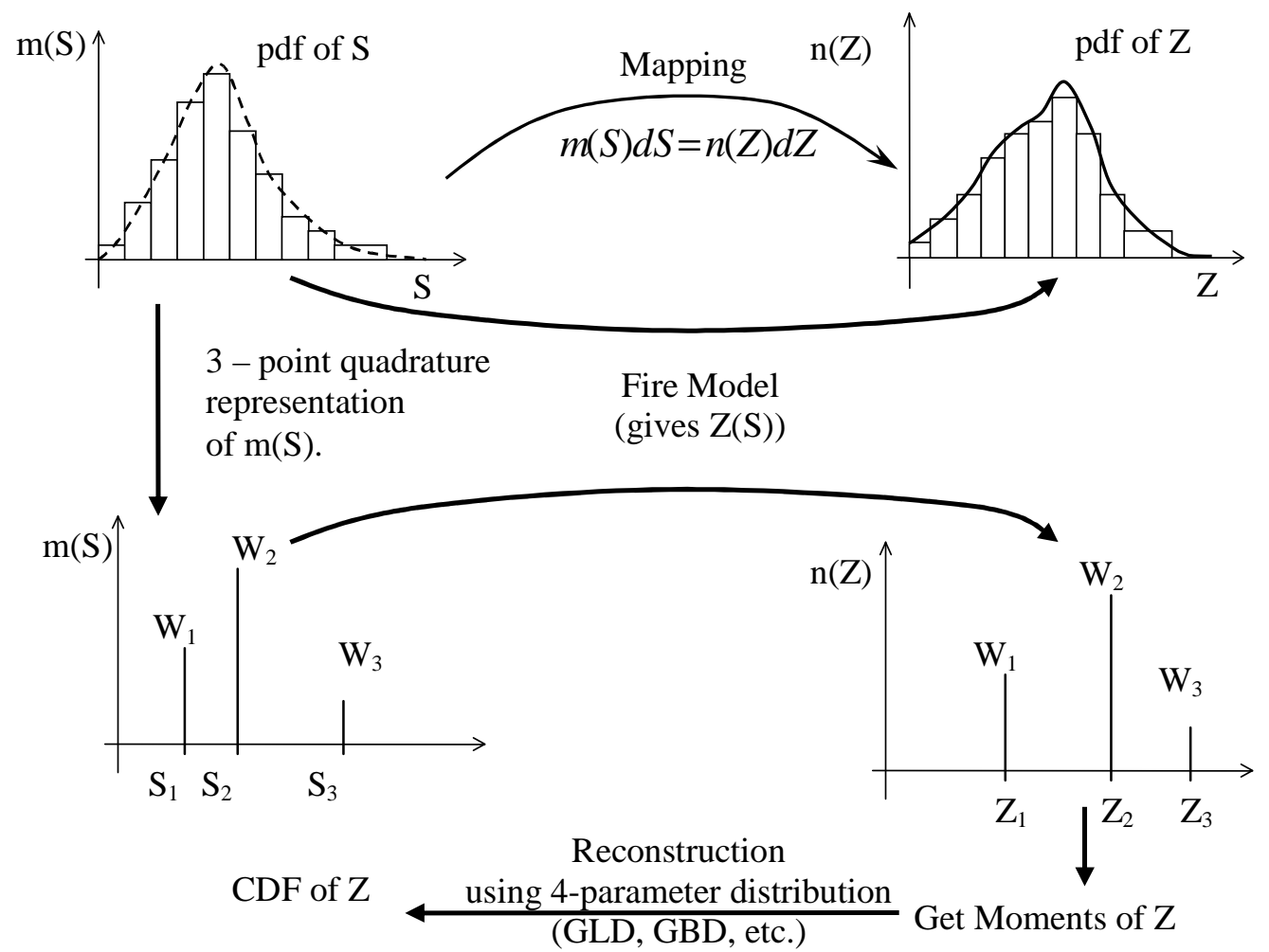

Fig. 2. 
DRAFT

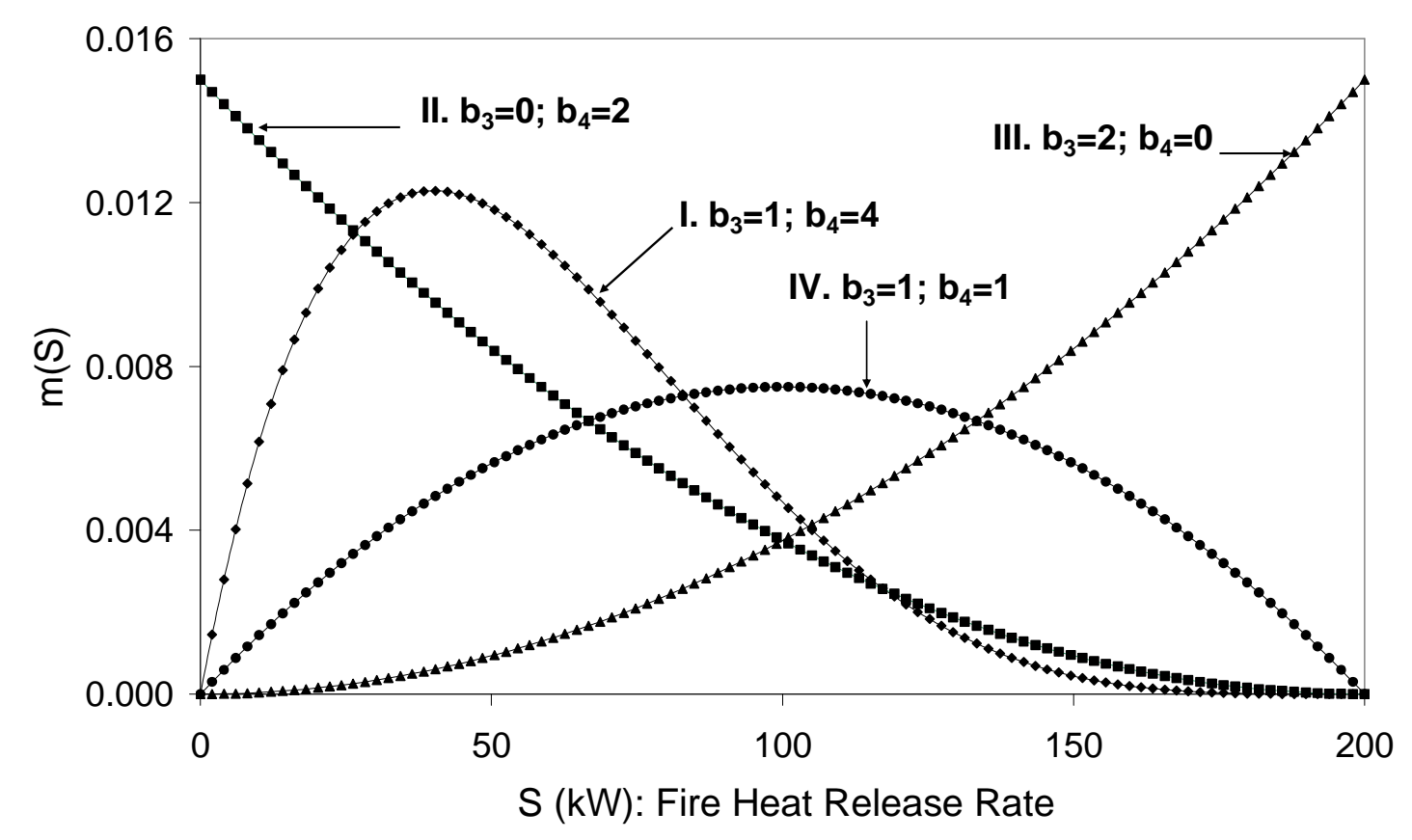

Fig. 3.

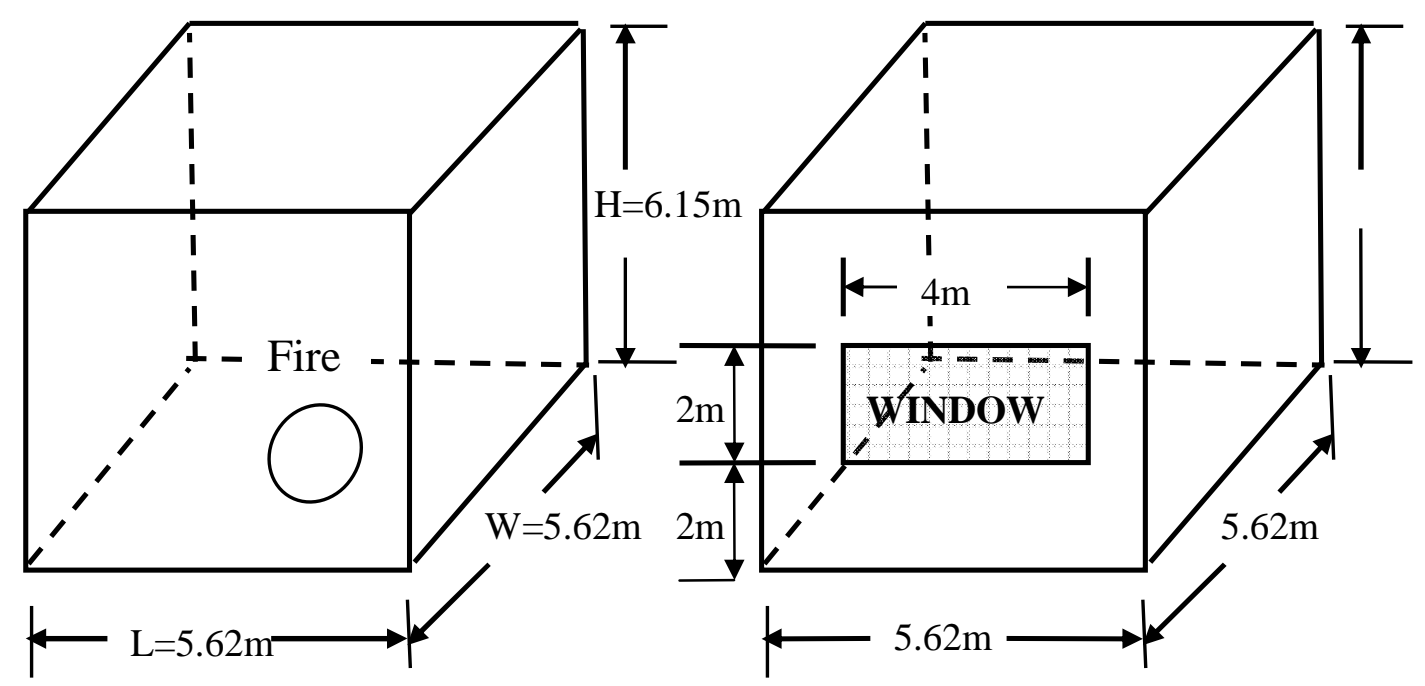

Fig. 4. 
DRAFT

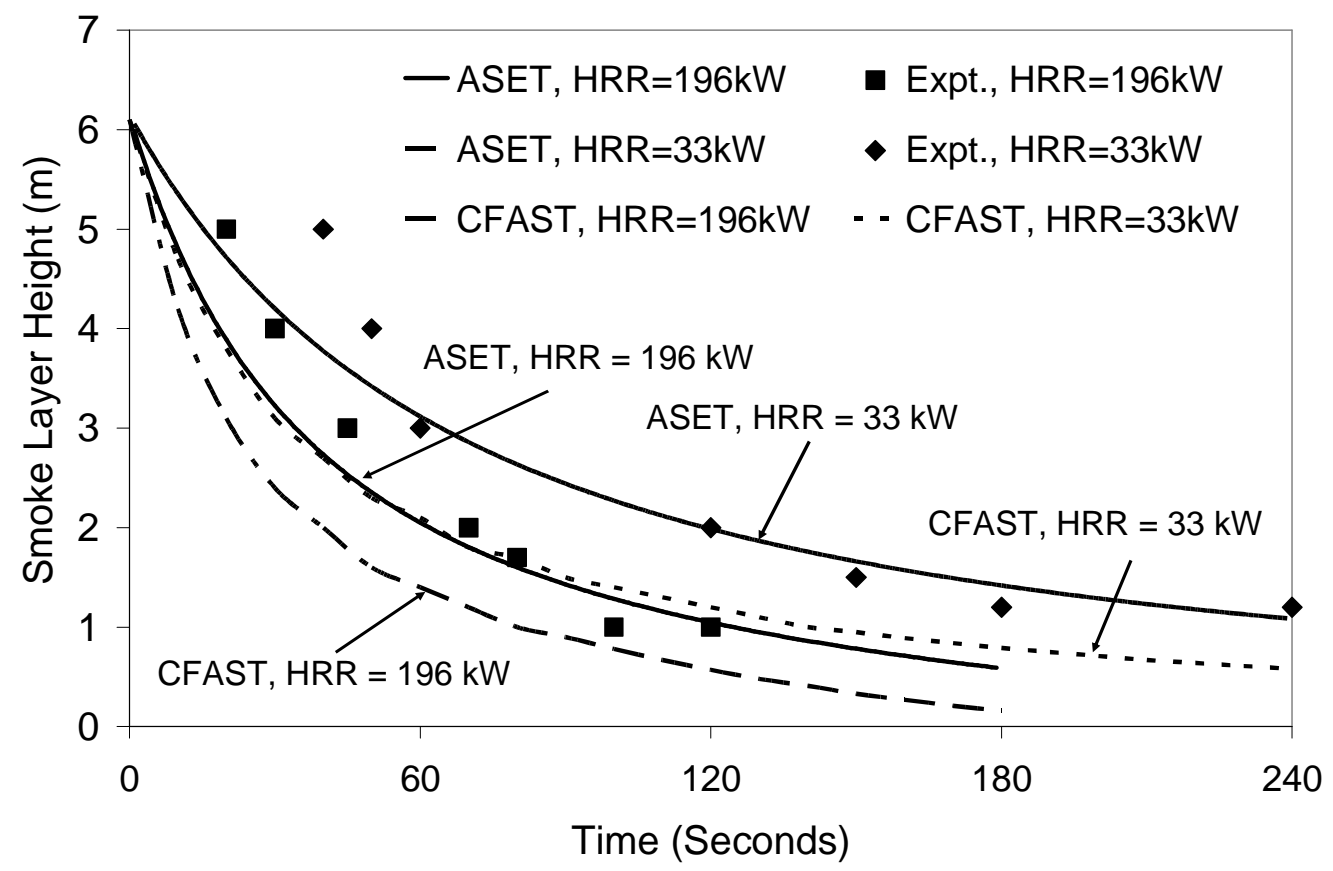

Fig. 5.

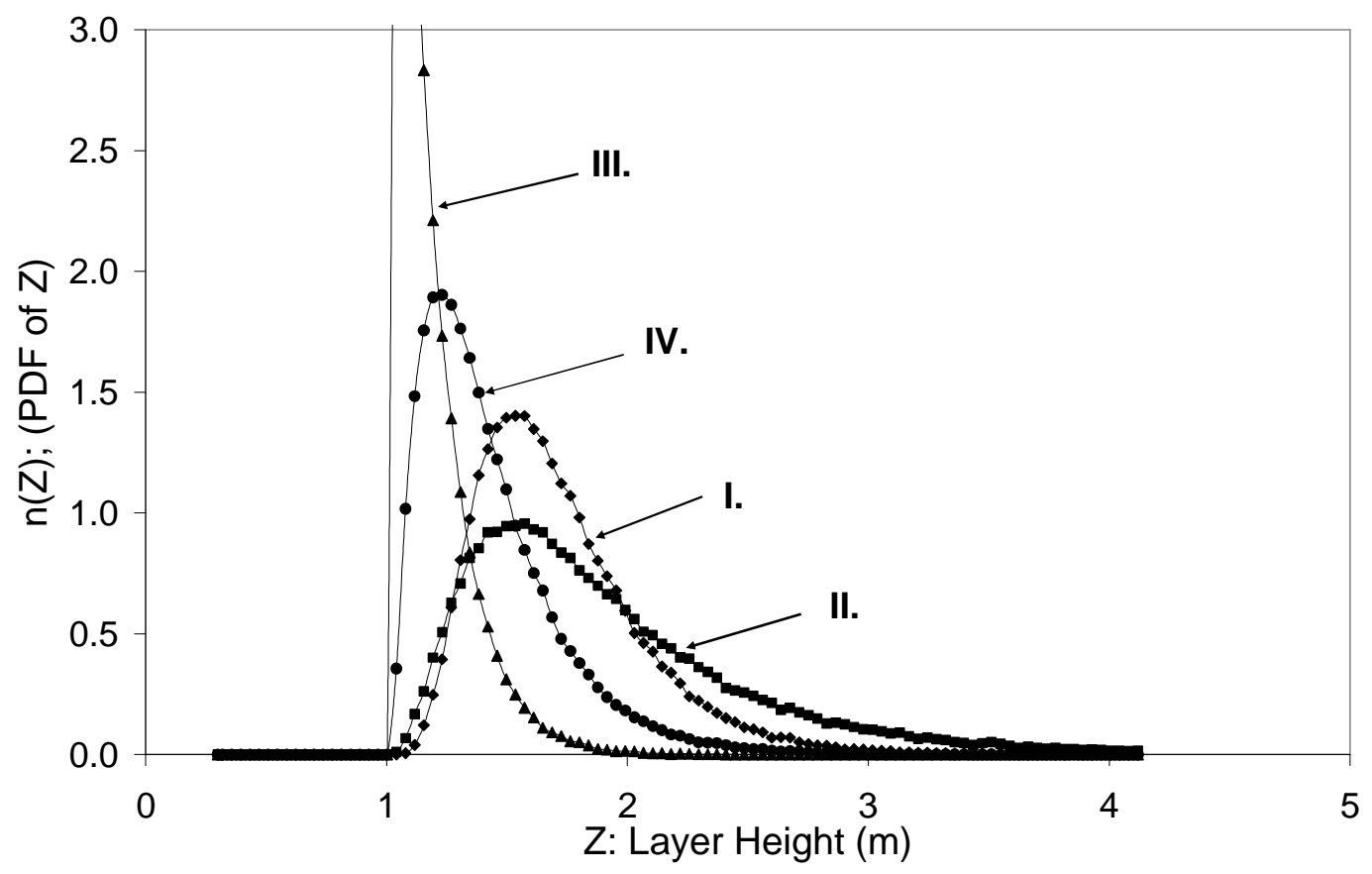

Fig. 6. 
DRAFT

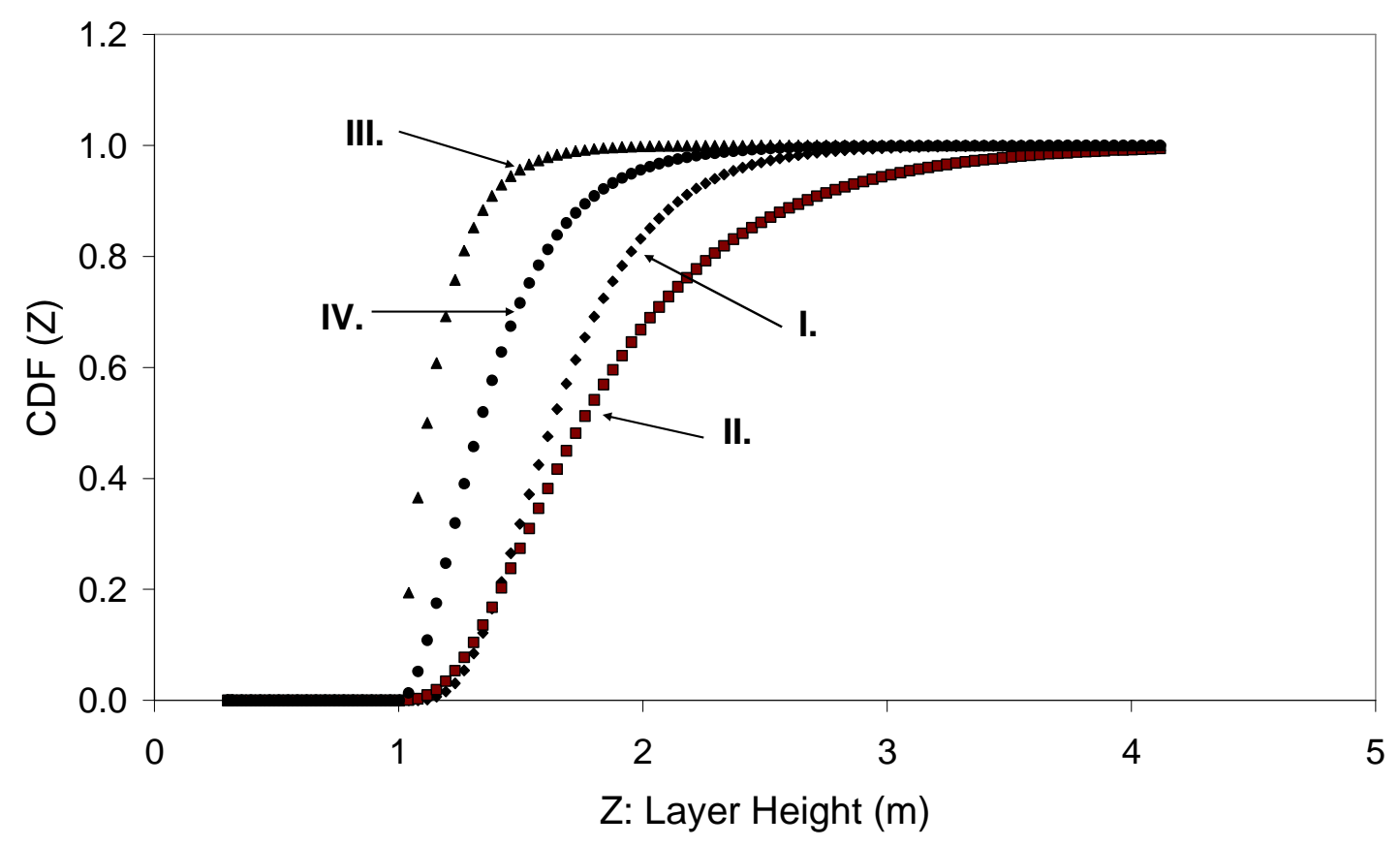

Fig. 7.

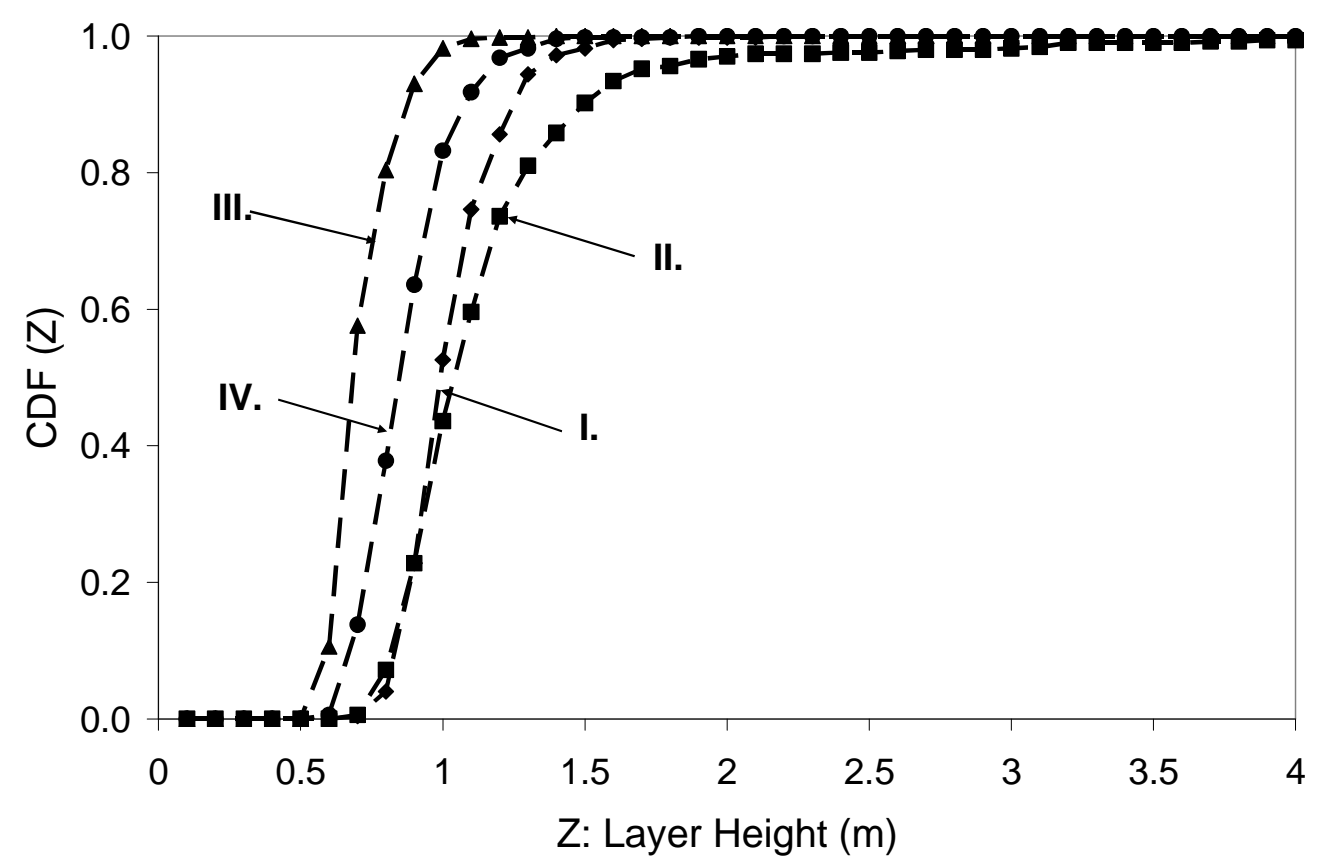

Fig. 8. 
DRAFT

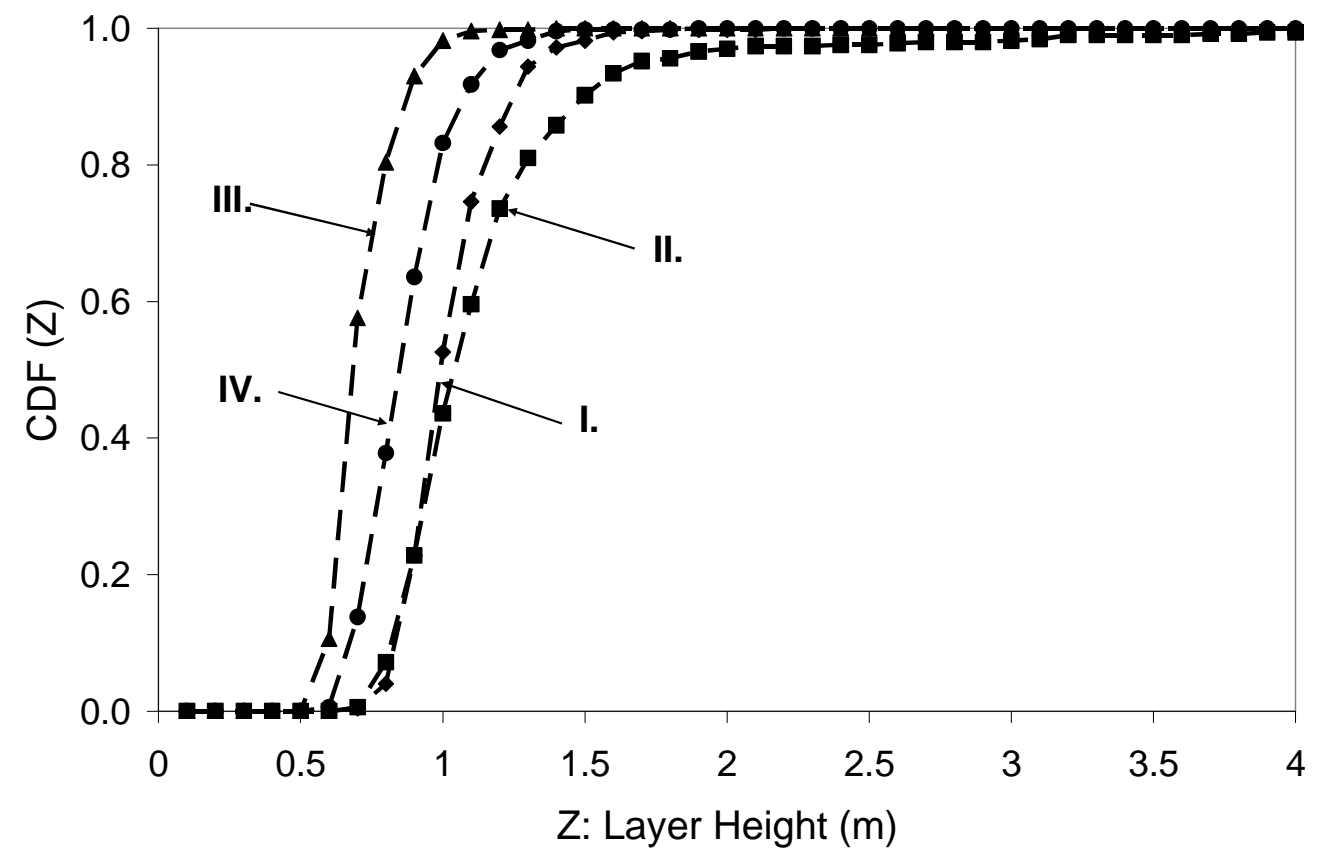

Fig. 9.

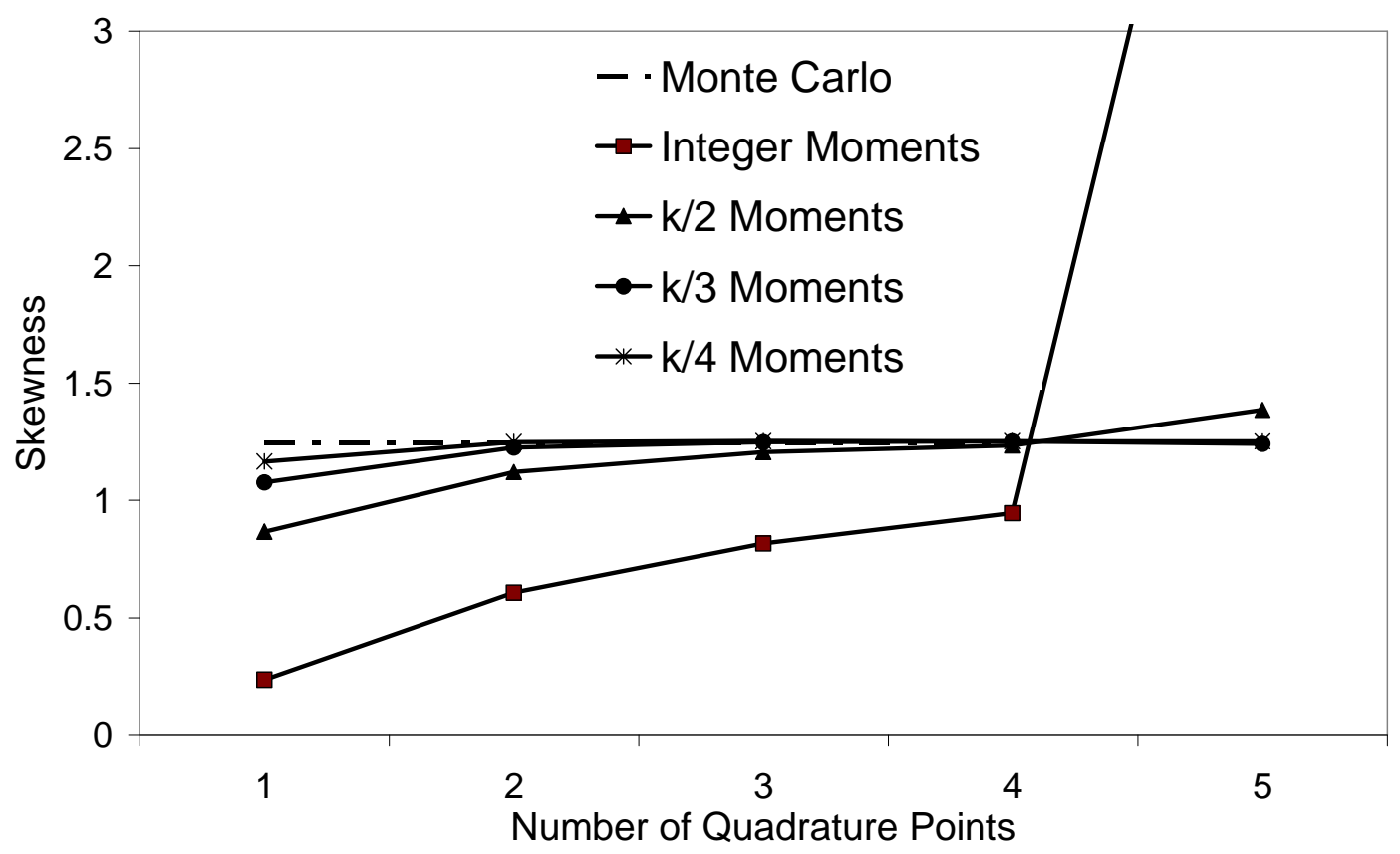

Fig. 10. 
DRAFT

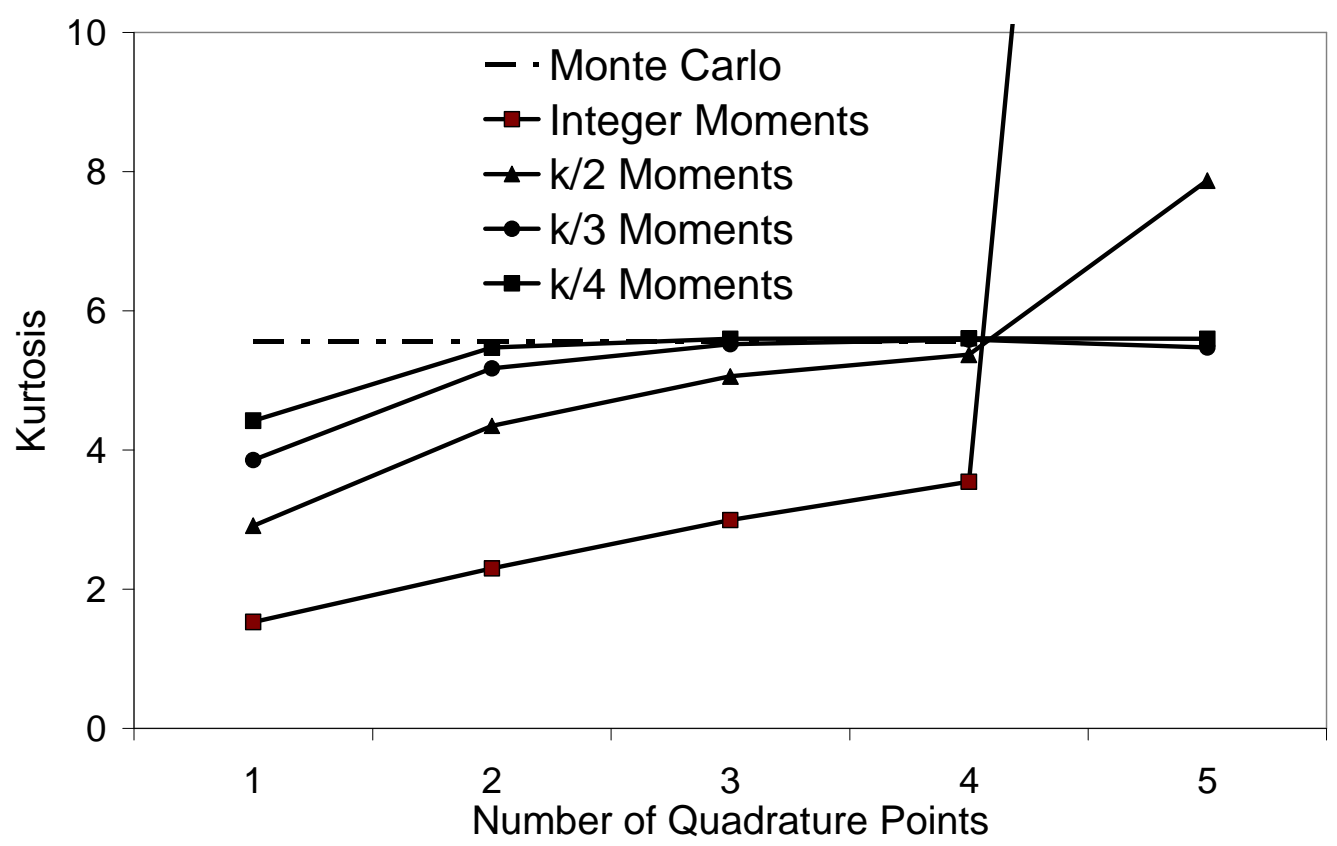

Fig. 11.

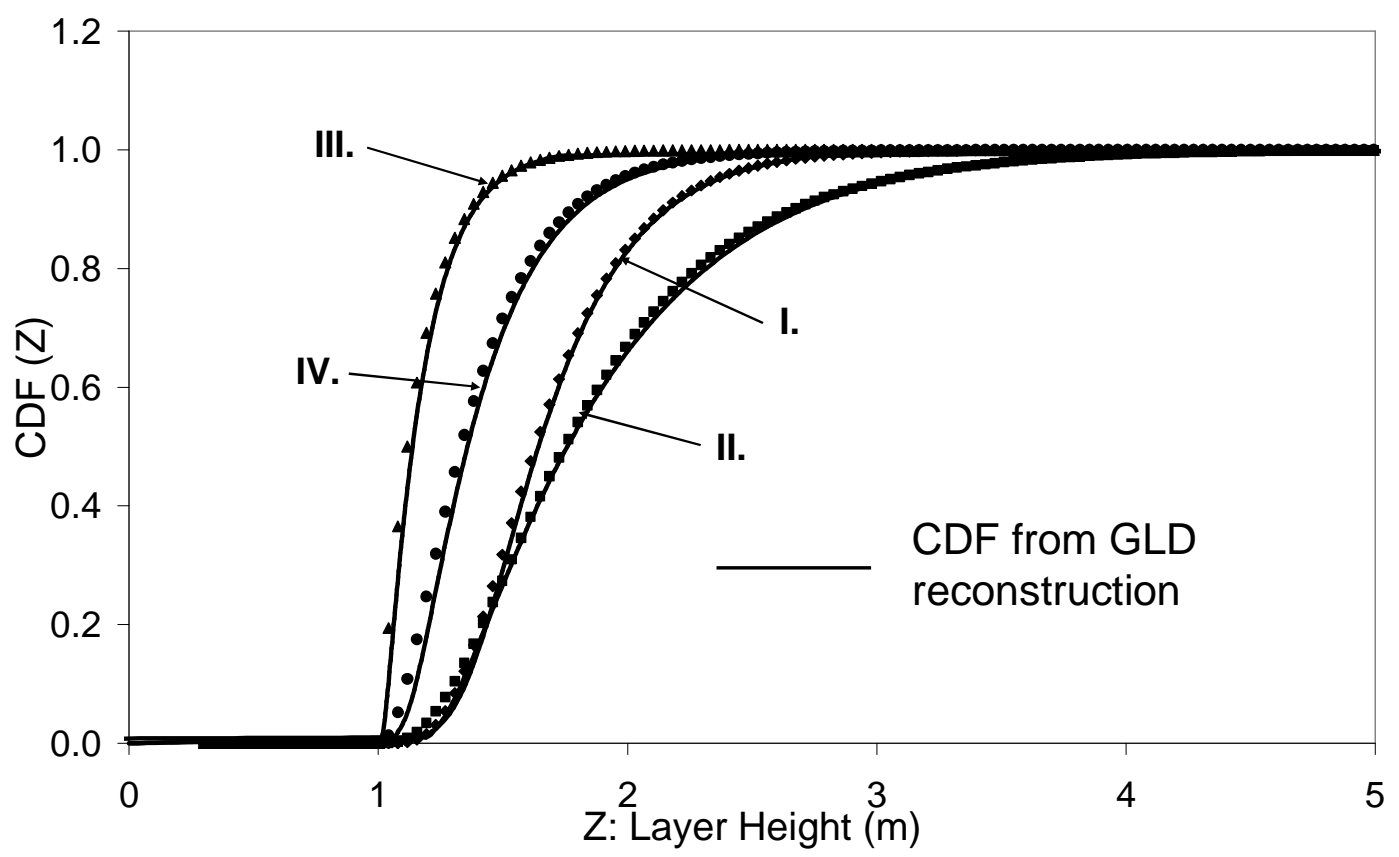

Fig. 12. 
DRAFT

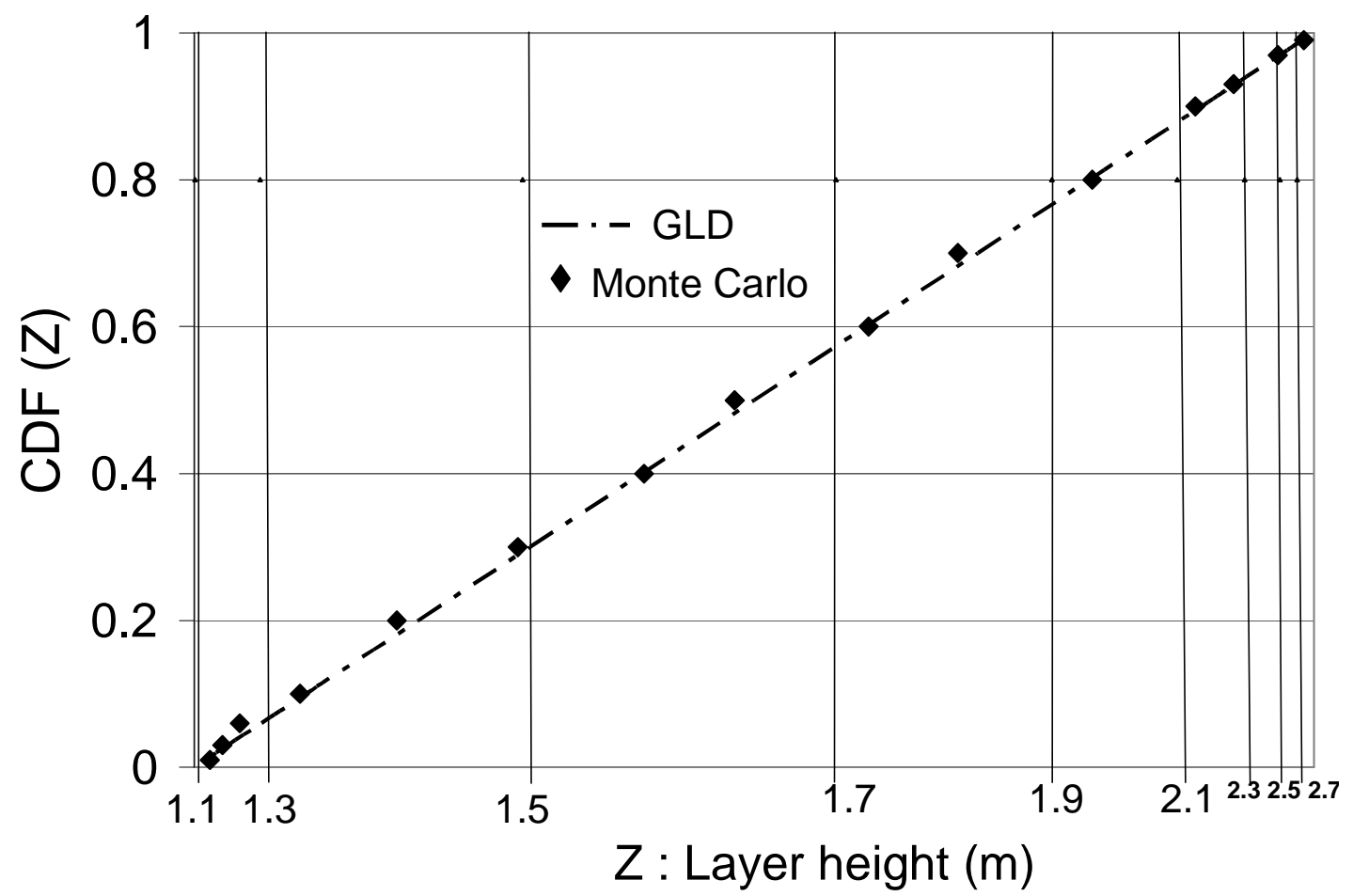

Fig. 13.

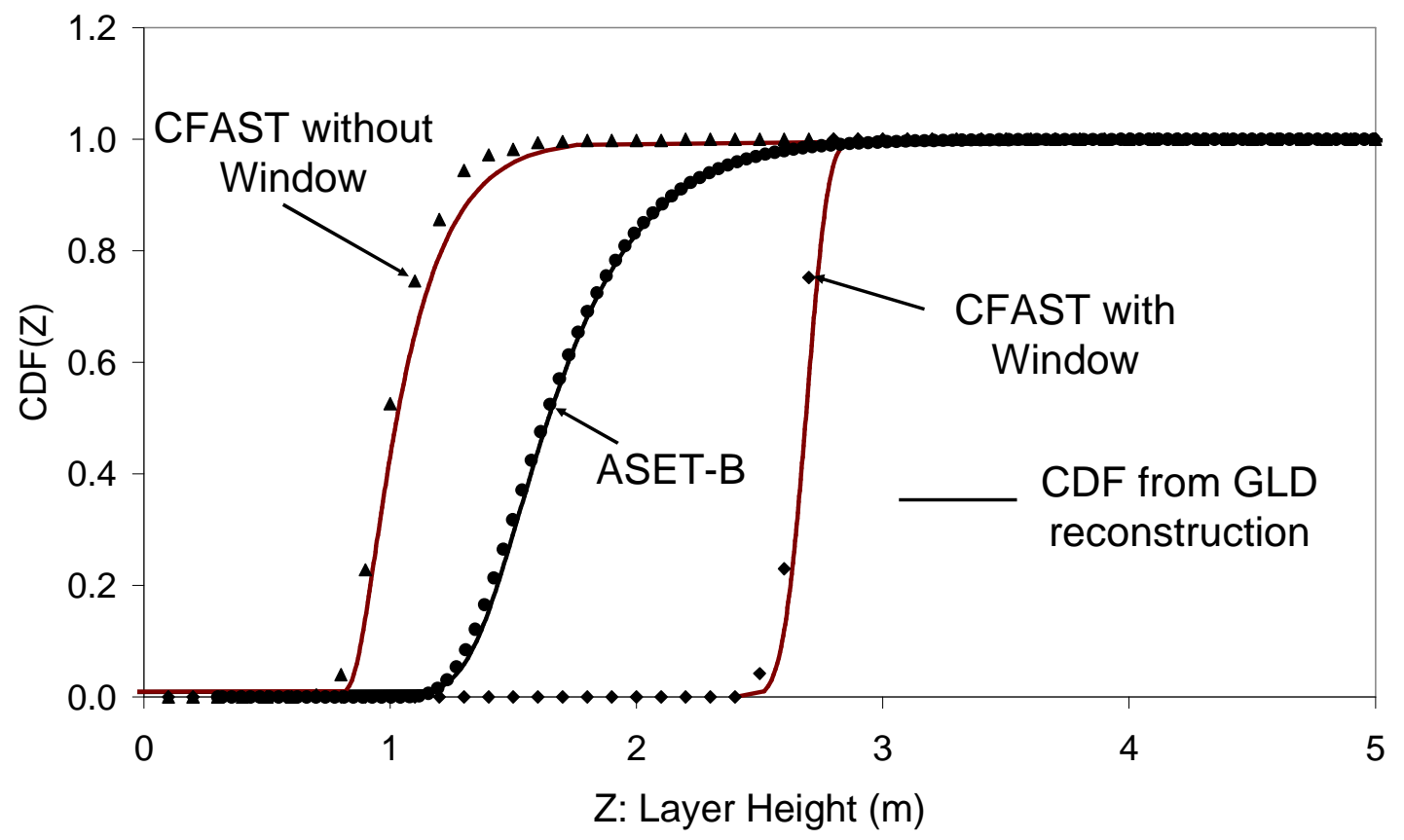

Fig. 14. 